\title{
INFLUENCE OF A STRUCTURE ON SYSTEM'S DYNAMICS ON EXAMPLE OF BOOLEAN NETWORKS
}

\author{
Olga V. Kirillova \\ Theoretical Physics Department, St.Petersburg State University, \\ St.Petersburg, 198904, Russia
}

\begin{abstract}
In this work we study the Boolean Networks of different geometric shape and lattice organization. It was revealed that no only a spatial shape but also type of lattice are very important for definition of the structure-dynamics relation. The regular structures do not give a critical regime in the investigated cases. Hierarchy together with the irregular structure reveals characteristic features of criticality.
\end{abstract}

Keywords: Boolean networks, structure influence, Kauffman model

\section{Introduction}

In 1969 S. Kauffman purposed to envisage a partial case of cellular automata (CA) for investigation of biological processes [1]. It was found that subclass of CA called Boolean networks reveals a number of properties successfully interpreted in terms of gene networks of living organisms. After this the tool of Boolean networks became fairly useful for investigations of a large range of phenomena: in spin glass theory [2], gene and neural networks [3, 1, 4, 5], chaos controlling problem [7] etc. Thus the study of properties of such systems acquired the undoubt interest. It was found and investigated both numerically and analytically many characteristic properties and regularities of Boolean networks [1, 2, 6].

For random Boolean networks behavior it was revealed that there are three different phases of system dynamics: chaotic, ordered and marginal (which supposed to be critical) [1, 4, 5]. It was pointed out that the behavior of such systems depends on many factors. Let us stop at such characteristics of Boolean networks as its structure.

Investigations of the relation between a structure and dynamics in real, for instance, biological systems are extremely difficult. At least we want to understand one qualitativley. For example, by the means of representation 
of gene regulation as a net of units acting each other, which have only two possible states. In this case the tool of Boolean Networks is very convenient.

So we envisage several networks of different forms with distinctions in geometric shape as well as in action organization. Considering the evolution of such systems for different dynamic rules, the choice of which is resulted in obtained for random Boolean networks relations, which admit to put system dynamics into ordered, chaotic or marginal class, we try to understand how a structure influences on system behavior in different phases. The investigation of system behavior has been made by the means of analysis of measuring trajectories convergence in the phase space of system's states, in other words through the measuring of the Hamming distance $(H d)$ [.

We do not try to perform any rule classification for cellular authomata with a definit structure as it has been done in [9]. Let us stress that we investigate no only different lattice structures but also different geometric shapes of the Boolean Networks.

Section 2 of this paper defines Boolean Networks and describes the possible approach to its investigations. In section 3 the studied structuries and rules of dynamics are presented. Section 4 describes obtained results. Finally, section 5 gives some conclusions.

\section{Definitions and approaches}

Boolean Networks can be considered as an example of cellular automata [8, 12]. The theory of cellular automata is quite successfully applied to study complex systems. [10

A Boolean network can well represents a big set of very different structures of the system from random Boolean networks models introduced by Kauffman [1] with its totally random structure to cellular automata with a regular lattice and local interactions.

A Boolean network is represented by a system of $N$ interconnected elements with only two possible states 0 and 1 (on/off). Any element in the system can has a connection with $K$ elements, where $K$ can be varied for different units. Under term connection it is assumed that $K$ other elements influence the center element. According to a logical or Boolean rule every element is moved to the next state. A state of the system is defined as a pattern of states (on/off or 0/1) of all elements forming it. All elements are updated synchronously, moving the system into the next state, and each 
state can has only single resultant one. The total space of the system's states is defined as all possible $2^{N}$ combinations of the elements' values in the system. Since the number of all possible states is limited and transition rules are fixed and do not depend on time, the system reaches a limit cycle or a fixed point called an attractor. Attractors may be envisaged as the "target area" of an organism, i.e. the cell's type in the end of development. Limit cycles can be considered in certain aspects as biological rhythms [1, 11].

As was pointed out in studying of models on the basis of Boolean networks [6, 1], the behavior of such systems can be varied in a wide range from order to chaos and in number of cases, has quite nontrivial character.

Eventually the choice of topology or a structure of interactions when another parameters are fixed, one can consider as one of the means of controlling chaos [7].

Due to totally characterize behavior of the system, in other words to say to which trajectory every from $2^{N}$ points of phase space belongs, one need to get the phase portrait of space of states of the system, namely the number of attractors, basins of attractors, a size of a limit cycle, if a more detailed analysis is desirable the number (or percentage) of Eden gardens, the number of trees, a transient length etc are calculated. If the system size is big enough it is extremely difficult to do, therefore in notes about the system behavior generally, it is reasonable to be limited by the more rough characteristics as, for example, the Hamming distance. Let us take one as the main characteristics of system behavior (in terms chaos/order).

Hamming distance is a reasonable and often useful measure of distance in the configuration space of states of a binary system. It is defined as the number of bits which are different between the binary sequences $S_{1}$ and $S_{2}$. Usually normalized Hamming distance $(H d)$ is considered

$$
H d=\frac{1}{N} \sum_{i=1}^{N}\left(\sigma_{i}(t)-\sigma_{i}^{\prime}(t)\right)^{2}
$$

where $N$ - number of elements in the system, $\sigma_{i}(t)$ - the state of $i$-th element at the moment $t, S_{1}=\left\{\sigma_{i}\right\}_{1}^{N}, S_{2}=\left\{\sigma_{i}^{\prime}\right\}_{1}^{N}$.

Any configuration corresponds to the point in the space of all possible configurations. According to the rules of dynamics, in other words, system's evolution, each initial configuration traces out a trajectory in time. As it was revealed if the process is chaotic, the trajectories of nearby configurations diverge (in number of cases exponentially or as for instance, on lattices with 
discrete variables and nearest-neighbor interactions the Hamming distance increases with a power law) in time, if the system's dynamics is ordered then closed trajectories converge, in the critical case ones neither converge nor diverge, the distance between them preserves almost the same in time.

Let us consider pairs of configurations which differ only in a single site, i.e. initial $H d$ is equal to $1 / N$.

If we consider just two trajectories and on this basis make the conclusion about system behavior in a whole, it seems to be no quite correct. Thus due to get more adequate statistical picture we consider statistically avereged Hamming distance defined by the next means.

At first let's take a state, where only in a random single position stay the unit,

the next state is chosen so that difference from the former is only in the first position and calculate the convergence of these trajectories.

Further we calculate the convergence of the former trajectory and one, which differ from it only in $3 \mathrm{~d}$ position. And so far for all odd numbers.

So we investigate $\mathrm{N} / 2$ pairs of trajectories.

The next step. A state with couple of units in two random positions is envisaged and then the previous step is repeated.

And so far for states with $3,4, \ldots, N-1$ units.

Generically we have done uniform selection from the total space of states and investigated $\frac{N(N-2)}{2}$ pairs of trajectories.

In total we have the average $H d(t)$ defined as

$$
H d(t)=\frac{2}{N^{2}(N-2)} \sum_{\sigma, \sigma^{\prime} \subset \Omega} \sum_{i=1}^{N}\left(\sigma_{i}(t)-\sigma_{i}^{\prime}(t)\right)^{2}
$$

where $\Omega$ is the set of trajectories choosen by suggested above means.

\section{$3 \quad$ Structures and rules of dynamics}

We have considered seven two-dimensional structures with connectivity $K=3$.

We tried to cover more different structures both in the sense of a spatial shape (ribbon closed to circle, torus, sphere, cone) and in the sense of links' organization (regular lattices, loops, cascades or hierarchy structures, feedback and autoregulation). In striving to answer the question, how the 
type of lattice, the spatial organization, boundaries, autoregulation, hierarchy influence the behavior of a system and how the structure and dynamic's rules interact, we used two sorts of rules namely, homogeneous (the same for all elements) and heterogeneous (one for odd and another for even elements). The choice of the rules in the model is reasoned by the fact revealed early for random Boolean networks ones, that the average connectivity of a network and rules governing its behavior are related by the next formula $K_{c}=\frac{1}{2 p(1-p)}$. As it was obtained if $\langle K\rangle<\frac{1}{2 p(1-p)}$ (where $\langle K\rangle-$ the average connectivity, $p$ - the bias of the rule of dynamics) dynamics have to be ordered, if $\langle K\rangle>\frac{1}{2 p(1-p)}$ we have to get chaos and if $\langle K\rangle=\frac{1}{2 p(1-p)}$ the behavior is critical [5, 13]. We use the same rules for all investigated structures.

- The first subject of our investigation is a ribbon closed to a circle. The elements are arranged on the edges of the ribbon. Each of them has links with its left and right neighbors and with the element opposite to it on the other edge of the ribbon.

- The second system has the structure represented by the directed graph, namely the binary tree where neighbors of an element are two successors for it nodes plus autoregulation (i.e. an element is itself neighbor). The last layer is closed in a circle. So we have the hierarchical model on a cone with autoregulation.

- In the third case we investigated the same but undirected graph. Neighbors of an element are two successors for it nodes plus its ancestor, in other words, here undirection excepts autoregulation. Thus we have a cone without autoregulation.

- The fourth investigated structure is represented by the regular honeycomb lattice closed in a torus.

- The fifth case contains the same as in the previous case lattice but here the boundaries' elements are selfregulated.

- In the sixth case we have the same lattice forming a sphere.

- The seventh case is represented by the regular squared lattice closed in a torus where neighbors of an unit are the left and the right and one above it. So we have a closed cascade of hierarchical regulation. 
Our rules are:

a. the homogeneous rule - if all three element's neighbor are switched on then the element will be switched on and it will be switched off in any other case. In the number presentation it is $01111111(p=0.125)$ (Here and below 0 in the rule means on and 1 means off. Each figure corresponds to the neighbor's state, where ones are situated from all 0 to all 1

$0 \begin{array}{llllllll}0 & 0 & 0 & 0 & 1 & 1 & 1 & 1\end{array}$

$0 \begin{array}{llllllllll}0 & 0 & 1 & 1 & 0 & 0 & 1 & 1\end{array}$

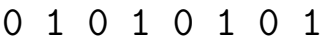

- - - - - - - -

$\left.\begin{array}{llllllll}0 & 1 & 1 & 1 & 1 & 1 & 1 & 1\end{array}\right)$

b. here we have the same rule as in the point $\mathbf{a}$ for even elements and the next for odd ones - if all three element's neighbor are switched off then the element will be switched on and it will be switched off in any other case. In the number presentation it is the same as above plus $10000000(p=0.125)$

c. an element will be switched on only in case both 1 st and $2 \mathrm{~d}$ its neighbor or $3 \mathrm{~d}$ have such state. In the number presentation it is $01000010(p=0.25)$

d. here we have the same rule as in the point $\mathbf{c}$ for even elements and for odd ones - if 1st or both $2 \mathrm{~d}$ and $3 \mathrm{~d}$ element's neighbor are switched on then the element will be switched on and it will be switched off in any other case. In the number presentation it is the same as above plus $00011000(p=0.25)$

e. an element will be switched on only in case any pair of its neighbors has such state. In the number presentation it is $00010110(p=0.375)$

g. here we have the same rule as in point $\mathbf{e}$ for even elements and for odd ones - if only a single of element's neighbor is switched on then the element will be switched on and it will be switched off in any other case. In the number presentation it is the same as above plus $01101000(p=0.375)$

\section{Results}

We pay attention to the next characteristics : percentage of convergence couple of trajectories in the phase space $p c$, maximum pattern difference between them $m p$ and behavior $H d(t)$ (it is defined as period of the function). The data of percentage convergence are presented in table 1, maximum pattern difference and character of behavior $H d(t)$ - in table 2 and table 3 correspondingly.

Let us make some explanatory notes to the table 3. After a finite number of steps the system comes to a stationary regime that can be either a fixed 
point or a limit cycle. So when we follow the evolution a pair of trajectories one can observe that after a number of steps if the trajectories have not converged the Hamming distance is constant or some periodic function of time, moreover the period can be very large, that point out that at least one trajectory belongs to a large limit cycle, that is usually proposed by a feature of chaos. It is true for each pair of trajectories, therefore the bigger the period of $H d(t)$ is the more chaotic system behavior supposed to be. A number of iteration steps before trajectories have converged one can consider as the maximum transient length.

As it is easy to see in a whole for different structures the results vary. But in spite of this there is a general tendency in systems' behavior and several fluctuations $(1 \mathbf{b}, 3 \mathbf{c}, 1 \mathbf{d}, 7 \mathbf{d}, 6 \mathbf{g})$. For all $\mathbf{a}$ and $\mathbf{e}$ cases percentage of convergence is always very high $(100 \pm 0.0004$ for a case and $99.93 \pm 0.37$ for e one). Excepts second structure the maximum patterns difference is always more than $19 \%$ for a case and it is always less than $1.5 \%$ for $\mathbf{e}$ one. These cases correspond to the ordered behavior.

Qualitatively g case always demonstrates chaotic behavior (too large period of $H d(t)$, quite low the convergence percentage). Except second structure the maximum patterns difference is always more than 48. At the same time the character of $H d(t)$ behavior is very variable.

Case $\mathbf{b}$ has very low the maximum patterns difference (less than 1\%) for all structures and the results are close to each other. Majority of structures in this case demonstrate rather chaotic behavior.

The system behavior is analogous for all $\mathbf{c}$ cases excepts third structure. One can put it into the category of chaos.

In $\mathbf{d}$ cases besides the second and 7 th structures we have ordered behavior.

Overall, in our case the obtained results do not confirm those for random Boolean networks. These results allow to conclude that heterogeneity/homogeneity of rules influences on system's behavior in more extent than the $K, p$ relation. In boundary cases $(\mathbf{a} \mathbf{b}$ and $\mathbf{e} \mathbf{g})$ heterogeneity result in chaotic dynamics, homogeneity - to order one and vice versa for middle cases (c d). Let us stress that ordered dynamics in a and e cases has not special situations.

Let's stop at some interesting results and distinctions.

The case $1 \mathrm{~g}$ has the minimum percentage of convergence. Eventually in this case there are big number of attractors, large pattern difference between them. We stress that in spite of the high convergence percentage $1 \mathbf{d}$ case reveals obvious chaotic features, if trajectories do not converge the difference 


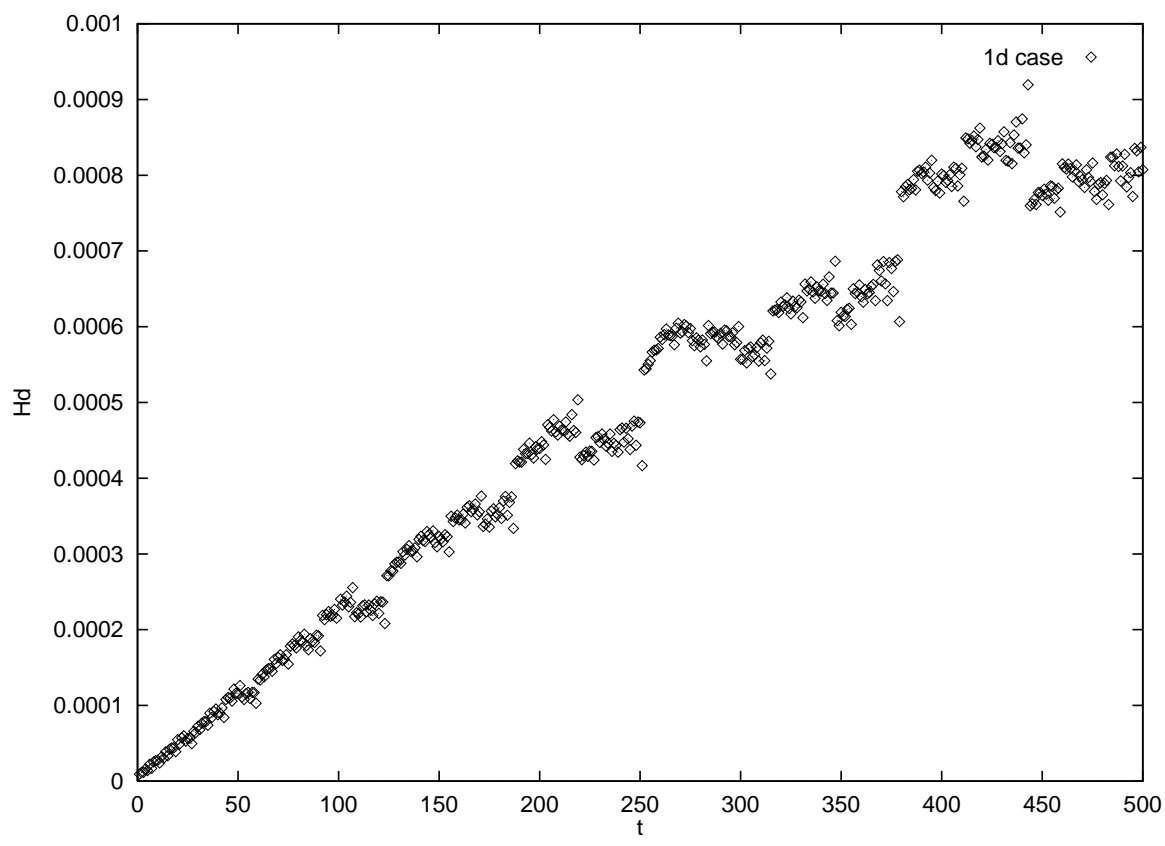

Figure 1: $H d(t)$ for the $\mathbf{d}$ rule on the 1st structure. Size of the system 1024.

between them becomes quite big. The character of $H d(t)$ is similar to $1 \mathbf{g}$ case (Fig. 1), (Fig. 2).

As for the second structure, here we observed some critical features in behavior of the system, especially in the $\mathbf{b}$ and $\mathbf{d}$ cases. For the $\mathbf{g}$ case we obtain that $H d(t)$ has period equal to 8 , percentage of convergence is quite high for chaotic behavior (Fig. 3). Overall this structure has no large maximum pattern differences, moreover it has the smallest value for both a and $\mathbf{g}$ cases and the smallest average.

In case of the third structure we can see strongly different from all other rules (from the same category) the result for the rule c. This case is characterized by too large (for rule c) convergence percentage and at the same time $H d(t)$ has quite large period (equal 12) (Fig. 4). Let us note that 3c case has the maximum pattern divergence equal to system's size. (It is the maximum among all other cases.) The minimum convergence percentage is observed not in $\mathbf{g}$ but in $\mathbf{b}$ case. $\mathbf{G}$ and $\mathbf{a}$ cases have the maximum pattern difference and the maximum average of this value (for its categories). In (Fig. 5) one can see the $H d(t)$ distribution for $\mathbf{g}$ cases on this structure.

Comparing 4th and 5th structures one can conclude that the influence of 


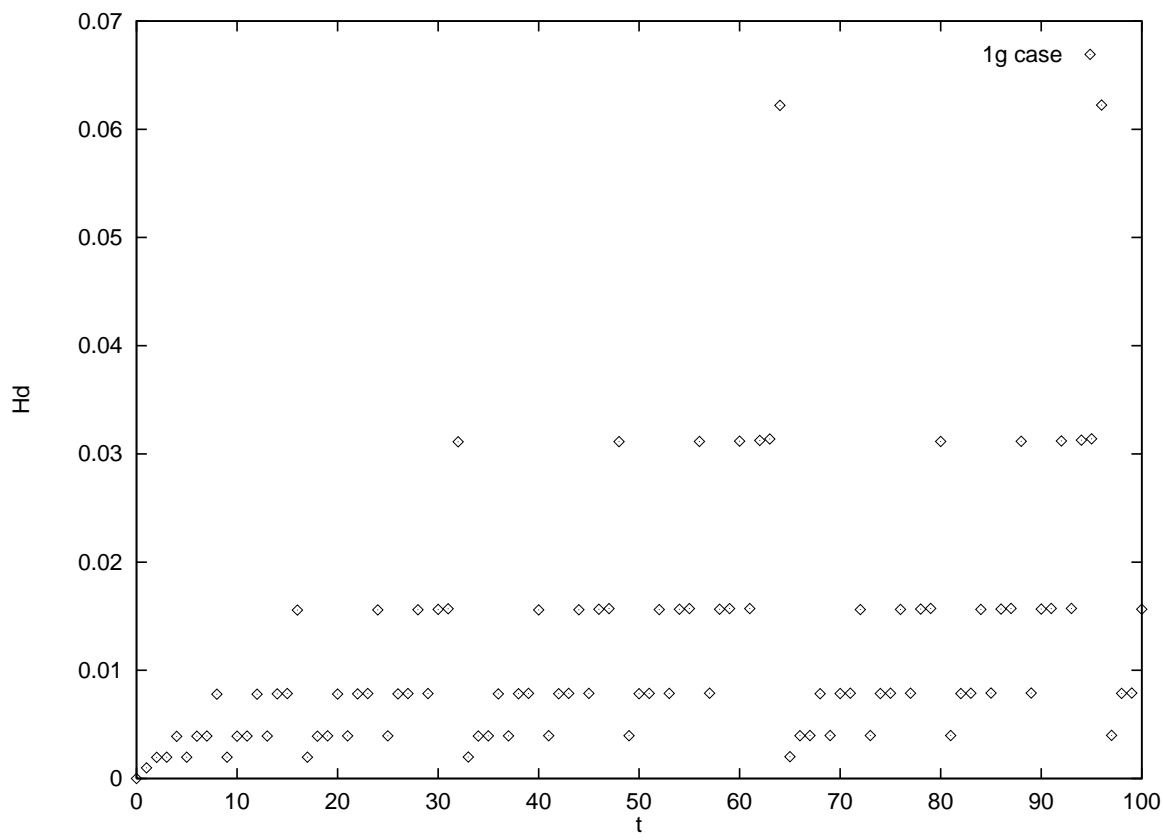

Figure 2: $H d(t)$ for the $\mathbf{g}$ rule on the 1st structure. Size of the system 1024.

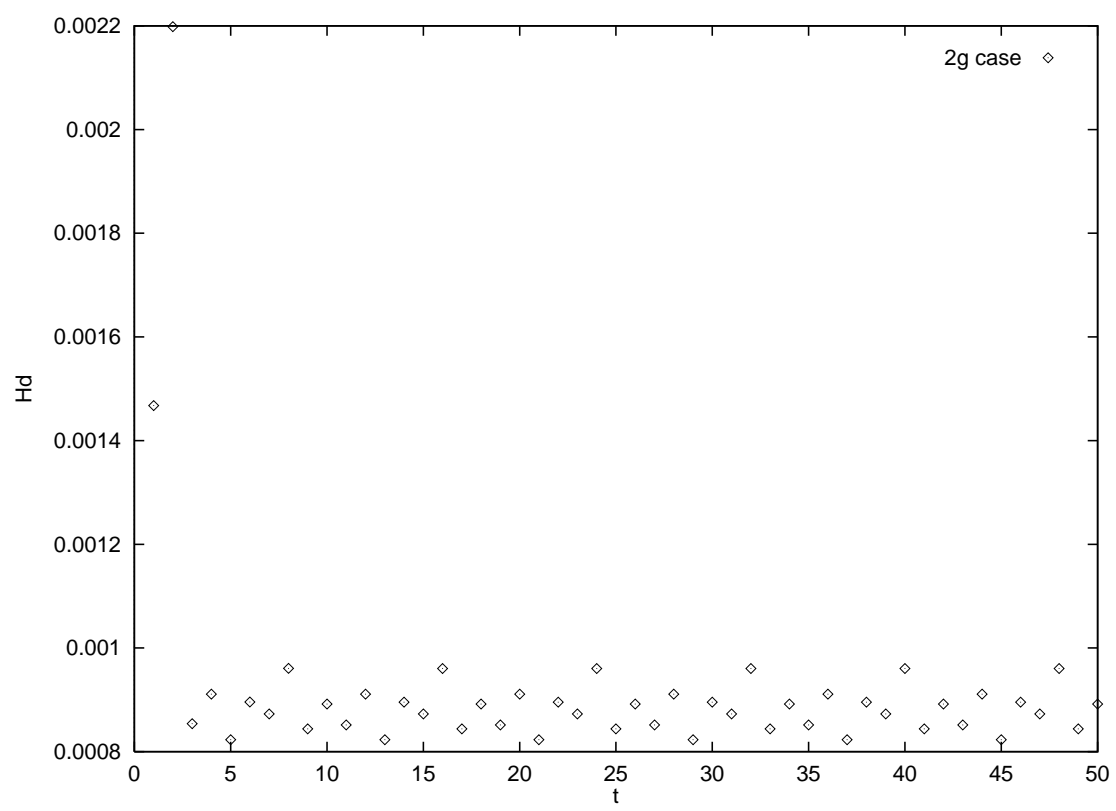

Figure 3: $H d(t)$ for the $\mathbf{g}$ rule on the second structure. Size of the system 1023. 


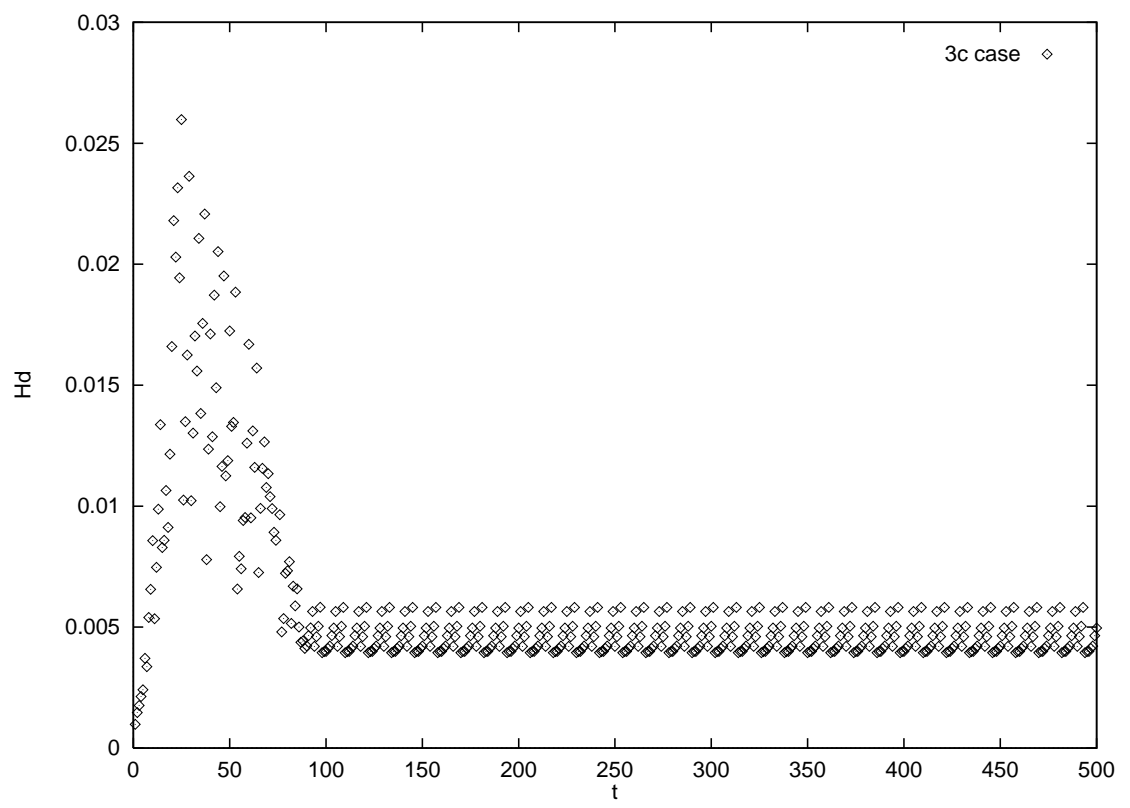

Figure 4: $H d(t)$ for the $\mathbf{c}$ rule on the third structure. Size of the system 1023.

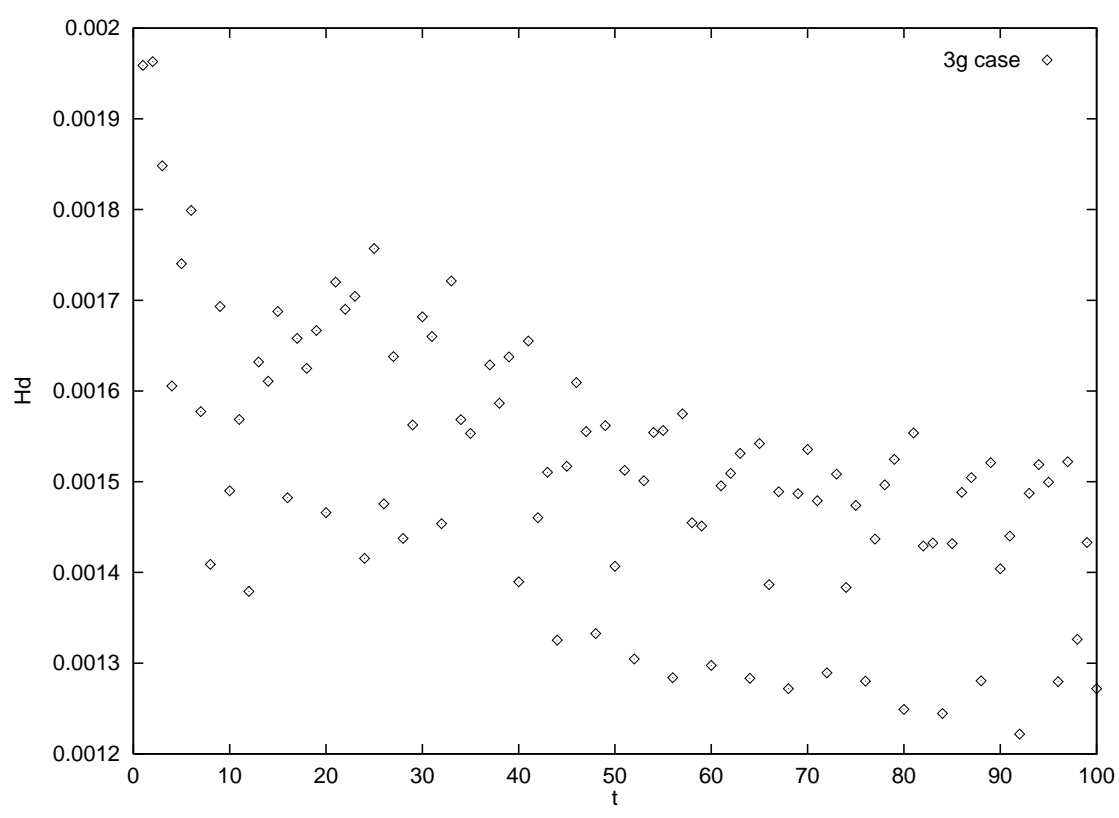

Figure 5: $H d(t)$ for the $\mathbf{g}$ rule on the third structure. Size of the system 1023. 


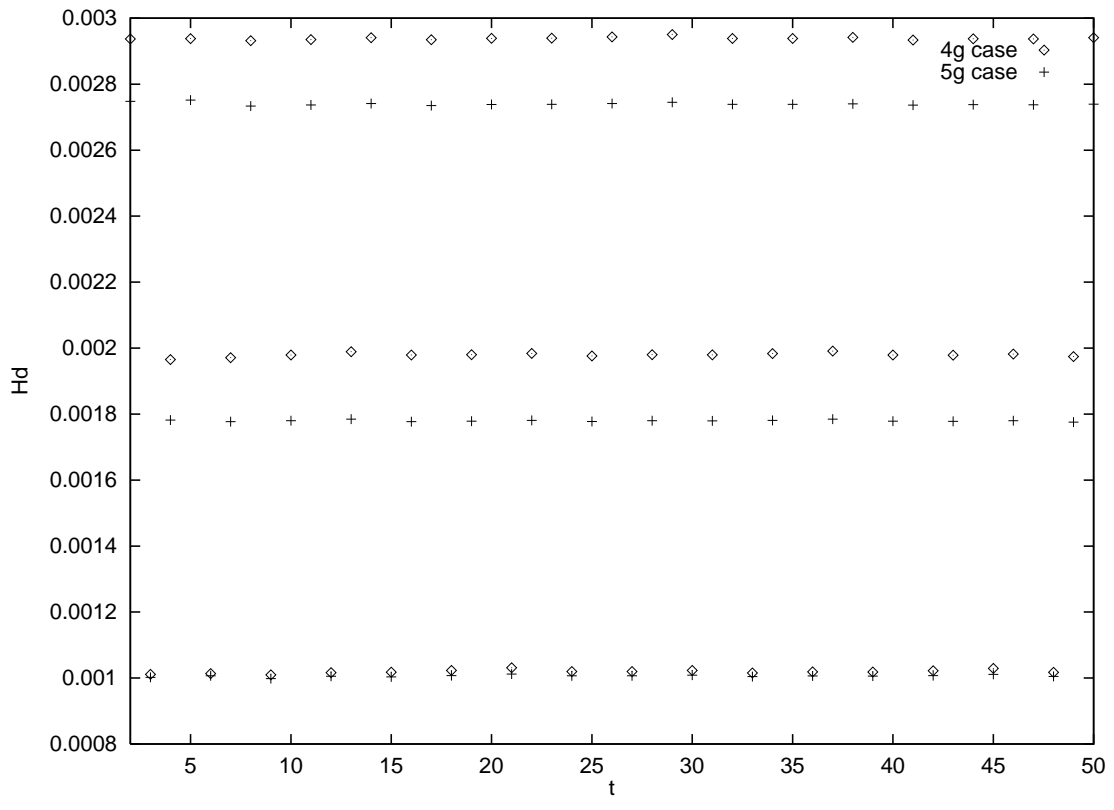

Figure 6: $H d(t)$ for the g rule on the 4 th (diamonds) and 5th (cross) structures. Size of the systems 1200 .

boundaries is negligibly small as is the one of the autoregulation. System's behavior is determined rather by the construction of the lattice. Here the behavior is either chaotic or ordered (not any critical features) with very strong differences between them. Excepting $\mathbf{b}$ and $\mathbf{g}$ cases the behavior on these structures is close to the 1st one. In (Fig. 6) one can see the $H d(t)$ distribution for $\mathbf{g}$ cases on these structures.

Behavior on the structure 7 is quite close to the 4 th and the 5 th ones excepting $\mathbf{d}$ case. This confirms a regular structure and a shape influence. The $\mathbf{7} \mathbf{d}$ case strongly differ from other (from its category) by low convergence percentage. In (Fig. 7) one can see $H d(t)$ distribution for $\mathbf{g}$ case on this structure.

The dynamics lead by the rules $\mathbf{b}$ and $\mathbf{g}$ in the most extent depends on the structure of interactions especially it is seen on the second, third and 6th structures, what allow to conclude that a spatial shape undoubtedly influence the dynamics of the system. But this influences is essential only for the definite rules. The $6 \mathrm{~g}$ case has too large convergence percentage for this rule. In (Fig. 8) one can see the $H d(t)$ distribution for the $\mathbf{g}$ case of the 


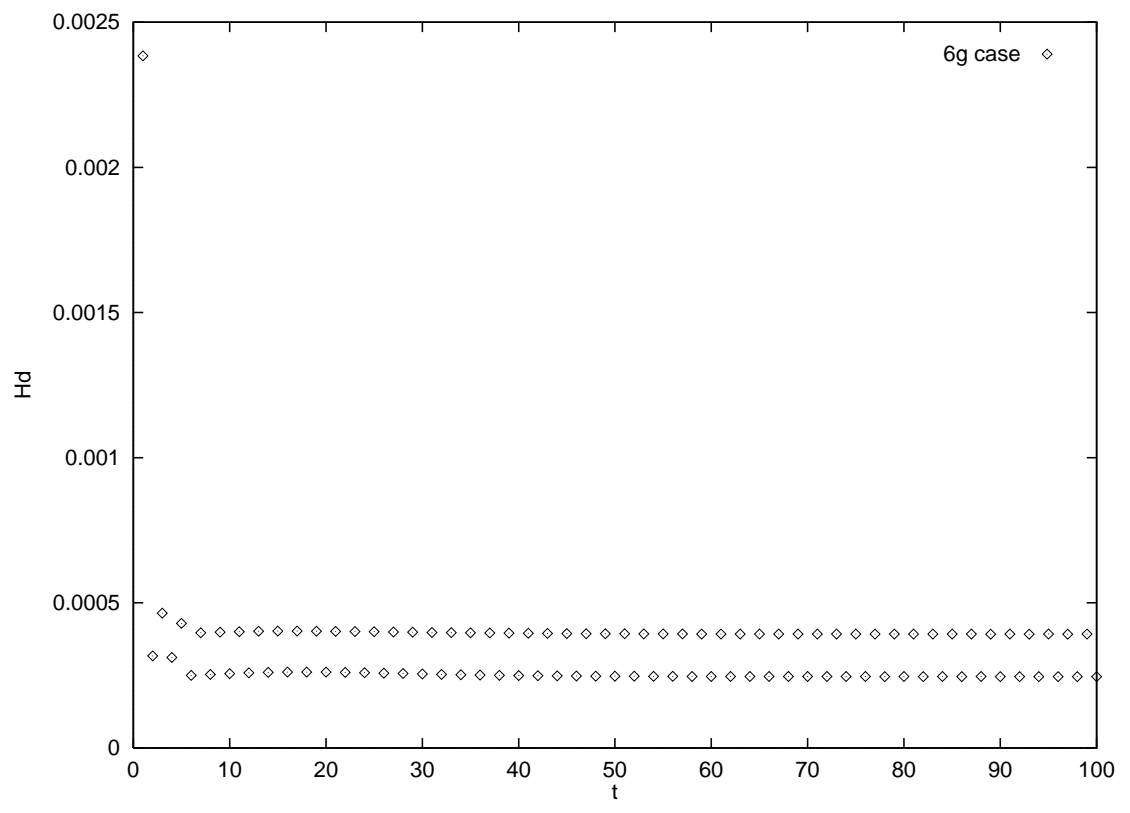

Figure 7: $H d(t)$ for the $\mathbf{g}$ rule on the 6th structure. Size of the system 1200.

6th structure. The minimum of convergence percentage is observed in the $\mathbf{c}$ case as well as for the second structure.

We studied the systems of different sizes. It was obtained that an increase of the system's size does not give large changes of dynamics, only slightly shifts percentage of convergence in the direction of the present tendency, i.e. for rather order cases it increases, for chaotic ones it decreases.

When we increase system's size, for the small $(<20)$ maximum pattern differences the results are the same and for large ones this value increases. The $\mathbf{a}$ and $\mathbf{g}$ rules have greater divergence what results in a more branched transient structure.

Also we considered other rules with the same bias for all structures.

It was revealed that the $\mathbf{a}$ and $\mathbf{e}$ cases with the same bias give results independent from the structure but strongly dependent on the rules, behavior can change from chaos to order. The maximum pattern difference is more stable than the convergence percentage. The cases with low convergence percentage are more stable to rules' changes. As for the rule $\mathbf{g}$ it gives the most robust result on the quality level. The same rules' changes result in different changes of system's dynamics for different structures. 


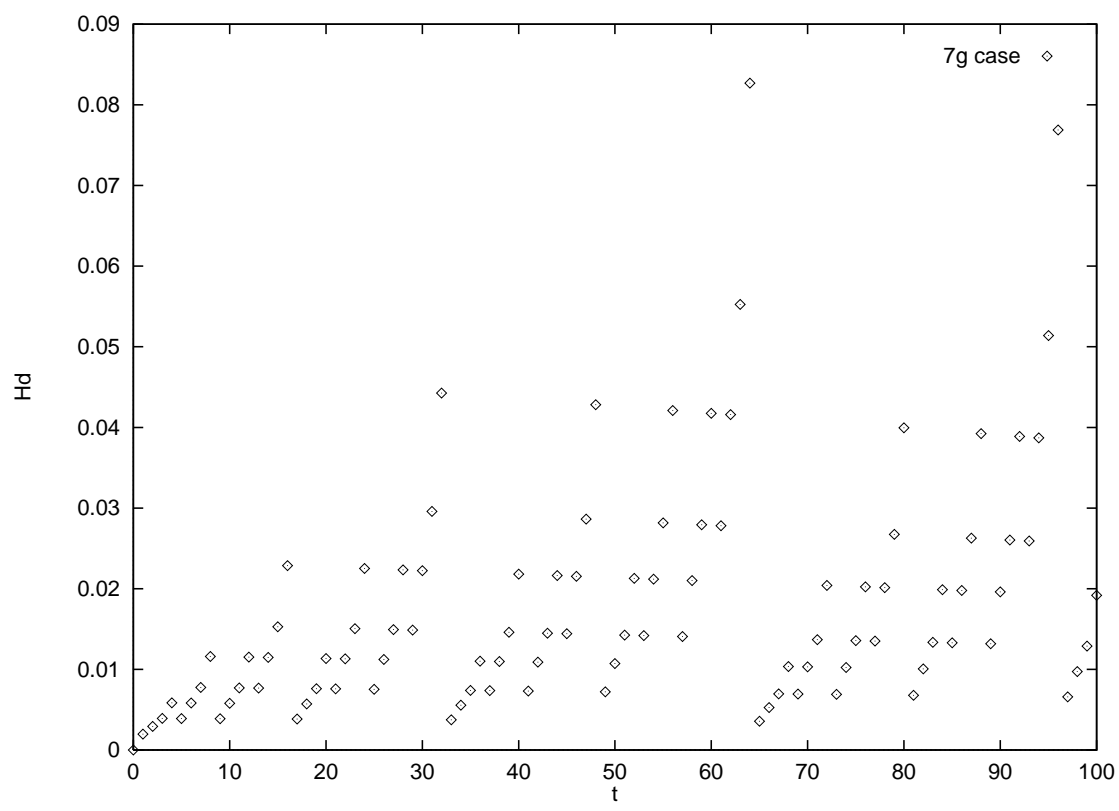

Figure 8: $H d(t)$ for the $\mathbf{g}$ rule on the 7th structure. Size of the system 1020.

\section{Conclusion}

This paper represents the investigation of influence of a Boolean network structure on behavior of the system. We have studied several different structures, which differ by the geometric shape, lattice organization and means of influence. We have used different rules of dynamics as well. The means by which the investigation has been done consists of the measuring of the averaged Hamming distance. In the work we used obtained for random Boolean networks $K, p$ relation and compared the results with ones in random Boolean networks models resulted in dividing of system behavior into ordered chaotic or marginal phases according to the $K, p$ relation.

Influence of the spatial shape as well as the organization of interactions on a lattice on system behavior is quite confirmed by the results obtained. As we saw both the second and the third structures form a cone but system's dynamics in these cases has strong distinctions, the second structure has the minimum average value of $m p$. At the same time the third structure is characterized by the maximum value of the corresponding parameter.

In the most extent the influence of the special shape of the network has been observed for the $\mathbf{b}$ and $\mathbf{g}$ rules. For the second, third and sixth structures 
percentage of convergence is essentially greater than for the other structures, that allows to conclude that behavior of Boolean Networks on a sphere is closer to a cone than to a torus or a closed ribbon.

The closed lattices themselves do not influence on system's behavior but in addition to hierarchy it decreases the convergence percentage, as it one can see on example of the 4,5,7 structures.

The influence of boundaries and adges is negligibly small.

Hierarchy in couple with irregularity gives the critical behavior $(2 \mathbf{b}, 2 \mathbf{d})$ or shifts the character of system's dynamics in this direction $(2 \mathbf{g})$.

The investigation of the second and the 7 th structures pointed out that there is no essential influence of autoregulation.

$K, p$ relation obtained for random Boolean networks [5, 13] has not been confirmed by the results of our investigation of the concrete structuries.

In the larger number of cases small changes of the interaction's structure do not give large changes of dynamics but in some cases, it is so.

Acknowledgment. The work was partially supported by State Committee of Russian Federation for high education grant No 97-14.3-58.

\section{References}

[1] Kauffman S.A., J. Theor. Biol. 22437 (1969)

[2] Derrida B., Flyvbjerg H., J. of Phys. A 19 L1003 (1986)

Derrida B., Flyvbjerg H., J. of Phys. A 20 L1107 (1987)

[3] Bornholdt S., Sneppen K., Phys. Rev. Lett. 81(1) 236 (1998)

Glass L., Hill C., Mestl T., Pacific Symposium of Biocomputing '98 (1998)

de Sales J.A., Martins M.L., Stariolo D.A., Phys. Rev. E 55(3) 3262 (1997)

Thomas R., J. Theor. Biol. 42563 (1973)

[4] Kauffman S.A., Physica D 42135 (1990)

[5] Luque B., Sole R.V., Europhys. Lett. 37(9) 597 (1997)

[6] Bastolla U., Parisi G., Physica D 115203 (1998)

Bastolla U., Parisi G., Physica D 115219 (1998) 
[7] Sompolinsky H., Crisanti A., Phys. Rev. Lett. 61(3) 259 (1988)

Glass L., Bagley R.J., Mestl T., Phys. Rev. Lett. 79(4) 653 (1997)

[8] Wolfram S., Rev. Mod. Phys. 55601 (1983)

[9] Gerling , Physica A 162196 (1990)

Stauffer D., J. of Phys. A 24909 (1991)

Makowiec D., Physica A 236353 (1997)

[10] Bagnoli F., Rechtman R., Rufto S. Phys. Let. A 17234 (1992)

Gacs P. J. of Comp. Sys. Sci. 32(1) 15 (1986)

Toffoli T., Margolus M., Cellular Automata Machines,( MIT Press, Cambrige, 1987)

[11] Thomas R., J.Theor. Biol. 73631 (1978)

Kauffman S., At home in the Universe (Oxford Univ. Press, Oxford, 1995)

[12] A. Turing Proc. London Math. Soc. 2 42, 230 (1936)

J.Von Neumann The general and logical theory of automata, coll. works, 5, (McMillan, New York 1951)

J.Von Neumann Theory of self-reproducing automata, A.W.Burks (Ed.), (University of Illinois Press 1966)

[13] Derrida B., Pomeau Y., Europhys. Lett. 1(2) 45 (1986)

Derrida B., Stauffer D., Europhys. Lett. 2(10) 739 (1986)

Bastolla U., Parisi G., Physica D 981 (1996) 


\section{TABLES}

Table 1: In this table the data of convergence percentage of the couple of trajectories in the phase space are presented. Sizes of the systems have order $10^{3}$ elements.

\begin{tabular}{|c|c|c|c|c|c|c|}
\hline & $\mathrm{a}$ & $\mathrm{b}$ & $\mathrm{c}$ & $\mathrm{d}$ & $\mathrm{e}$ & $\mathrm{g}$ \\
\hline 1 & 100 & 99.49 & 0.0441 & 99.54 & 99.56 & 0.00076 \\
\hline 2 & 100 & 49.95 & 0.08636 & 50.06 & 100 & 35.53 \\
\hline 3 & 100 & 32.8 & 66.4 & 100 & 99.997 & 36.17 \\
\hline 4 & 100 & 0.0765 & 0.428 & 99.9992 & 99.9976 & 0.00366 \\
\hline 5 & 100 & 0.08937 & 0.4247 & 100 & 99.9626 & 0.008 \\
\hline 6 & 100 & 13.995 & 0.413 & 100 & 99.982 & 80.053 \\
\hline 7 & 100 & 0.072 & 0.42 & 0.455 & 99.9973 & 0.0035 \\
\hline
\end{tabular}

Table 2: In this table the data of the maximum pattern difference between the couple of simultaneously considered trajectories are presented. Sizes of the systems have order $10^{3}$ elements.

\begin{tabular}{|c|c|c|c|c|c|c|}
\hline & $\mathrm{a}$ & $\mathrm{b}$ & $\mathrm{c}$ & $\mathrm{d}$ & $\mathrm{e}$ & $\mathrm{g}$ \\
\hline 1 & 500 & 4 & 3 & 508 & 6 & 97 \\
\hline 2 & 1 & 10 & 2 & 3 & 1 & 7 \\
\hline 3 & 842 & 6 & 1023 & 16 & 15 & 292 \\
\hline 4 & 510 & 4 & 10 & 7 & 11 & 49 \\
\hline 5 & 375 & 2 & 6 & 6 & 10 & 58 \\
\hline 6 & 230 & 9 & 7 & 11 & 11 & 84 \\
\hline 7 & 495 & 3 & 8 & 18 & 12 & 208 \\
\hline
\end{tabular}


Table 3: In this table the data of period of $H d(t)$ function are presented. Sizes of the systems have order $10^{3}$ elements. ( $\mathrm{pt} \equiv$ point, in other words the moment (time step) when all envisaged pairs of trajectores have converged)

\begin{tabular}{|c|c|c|c|c|c|c|}
\hline & $\mathrm{a}$ & $\mathrm{b}$ & $\mathrm{c}$ & $\mathrm{d}$ & $\mathrm{e}$ & $\mathrm{g}$ \\
\hline 1 & 0 from $256 \mathrm{pt}$ & 2 & 1 & $>50$ & 1 & $>50$ \\
\hline 2 & 0 from $9 \mathrm{pt}$ & 1 & 2 & 2 & 0 from $11 \mathrm{pt}$ & 8 \\
\hline 3 & 0 from $16 \mathrm{pt}$ & 6 & 12 & 0 from $21 \mathrm{pt}$ & 1 & $>50$ \\
\hline 4 & 0 from $101 \mathrm{pt}$ & 1 & 1 & 0 from $18 \mathrm{pt}$ & 2 & $>50$ \\
\hline 5 & 0 from $148 \mathrm{pt}$ & 1 & 1 & 0 from $13 \mathrm{pt}$ & 2 & $>50$ \\
\hline 6 & 0 from $39 \mathrm{pt}$ & 8 & 1 & 0 from $34 \mathrm{pt}$ & 2 & $>50$ \\
\hline 7 & 0 from $105 \mathrm{pt}$ & 1 & 4 & 4 & 3 & $>50$ \\
\hline
\end{tabular}

